\section{Pestizidbelastung als Ursache für die fixierte Atemwegsobstruktion?}

Alif SM et al. Occupational exposure to pesticides are associated with fixed airflow obstruction in middle-age. Thorax 2017; 72: 990 - 997

Gibt es einen Zusammenhang zwischen der beruflich bedingten Exposition mit Pflanzenschutzmitteln und der „fixierten" Atemwegsobstruktion? Dieser Frage ging ein australisches Forschungsteam um Sheikh M Alif in einer Studie mit 1335 Patienten nach.

Mehrere Bevölkerungsstudien haben Hinweise auf einen Zusammenhang zwischen der beruflich bedingten Exposition mit Pestiziden und der chronisch-obstruktiven Lungenerkrankung (COPD) gefunden. Allerdings war in diesen bisherigen Studien die Spirometrie lediglich vor der Bronchodilatation durchgeführt worden. Die Forscher um SM Alif hingegen prüften nun die Lungenfunktion nach der Bronchodilatation.

\section{Methoden}

In die Follow-Up-Erhebung der Tasmanian Longitudinal Health Study (TAHS) von 2002 bis 2008 waren 1335 Patienten eingeschlossen. Die Teilnehmer waren im Durchschnitt 44,8 Jahre alt $( \pm 0,8$ Standardabweichung), 51,8\% von ihnen waren männlich, $87,4 \%$ gingen derzeit einem Beruf nach und 25,1\% waren zum Zeitpunkt der Analyse Raucher. Die Forscher erhoben die spirometrischen Daten und die berufliche Vorgeschichte der Studienteilnehmer. Um den Grad der beruflich bedingten Belastung mit Pestiziden, Herbiziden, Insektiziden und anderen die Atemwege belastenden Agenzien wie Gasen, Abgasen und Stäuben zu bestimmen, nutzten die Studienautoren

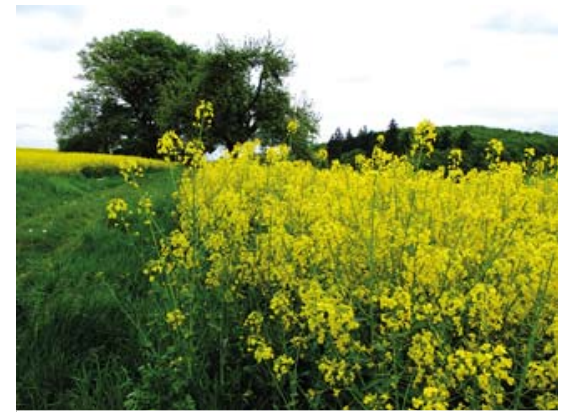

- Die berufliche Exposition mit biologischen Stäuben, Herbiziden oder Pestiziden ist laut Studie mit chronischen Atemwegserkrankungen assoziiert. Bildquelle: Renate Stockinger/Thieme Verlagsgruppe

die ALOHA + Job Exposure Matrix. Die fixierte Atemwegsobstruktion wurde anhand eines FEV1/FVC $<0,7$ nach Bronchodilatation und des unteren Normalwerts bestimmt.

\section{Ergebnisse}

In dieser australischen Studie war die mindestens einmalige Exposition mit biologischen Stäuben, Pestiziden und Herbiziden mit fixierter Atemwegsobstruktion assoziiert (relatives Risiko $[R R]=1,58$; 95\%-Konfidenzintervall [KI] 1,01-2,48 bzw. RR =1,74; 95\%-KI 1,00-3,07 bzw. $R R=2,09 ; 95 \%-K I 1,18-3,70)$. Auch zwischen der kumulativen Belastung in Jahren mit jeglicher Art von Pestiziden oder Herbiziden und der fixierten Atemwegsobstruktion konnte ein Zusammenhang festgestellt werden ( $R R=1,11 ; 95 \%-K I$ $1,00-1,25$ bzw. RR =1,15; $95 \%-K I 1,00-$ 1,32). Die Pestizidbelastung war zudem durchweg assoziiert mit chronischer Bronchitis und anderen Symptomen, die denen einer Atemwegsobstruktion entsprechen. Die mindestens einmalige Exposition mit Gesteinsstaub, Gasen/Abgasen und Dämpfen sowie Gasen, Stäuben oder Abgasen war nur bei Nicht-Asthmatikern mit einer fixierten Atemwegsobstruktion assoziiert.
FAZIT

In dieser Studie war jegliche Pestizidund Herbizidbelastung mit einer fixierten Atemwegsobstruktion und mit chronischer Bronchitis assoziiert. Zudem wurde ein Zusammenhang zwischen der Exposition mit biologischen Stäuben und einer fixierten Atemwegsobstruktion bei NichtAsthmatikern festgestellt. Die beruflich bedingte Exposition mit diesen Agenzien zu reduzieren, könnte dazu beitragen, die COPD-Belastung zu verringern, so die Autoren.

Kathrin Strobel, Mannheim 